\title{
Innovative Solar Tracking Concept by Rotating Prism Array
}

\author{
Héctor García, Carlos Ramírez, and Noel León \\ CIDYT, Monterrey Institute of Technology (ITESM), Avenida Eugenio Garza Sada 2501 South, Col. Tecnológico, \\ 64849 Monterrey, NL, Mexico \\ Correspondence should be addressed to Héctor García; hd.garcia.phd.mty@itesm.mx
}

Received 9 February 2014; Revised 25 June 2014; Accepted 26 June 2014; Published 20 August 2014

Academic Editor: Mahmoud M. El-Nahass

Copyright (C) 2014 Héctor García et al. This is an open access article distributed under the Creative Commons Attribution License, which permits unrestricted use, distribution, and reproduction in any medium, provided the original work is properly cited.

Solar energy has become one of the most promising renewable energies and is the most widely used nowadays. In order to achieve an optimum performance, both photovoltaic and solar thermal applications are required to track the position of the sun throughout the day and year in the most effective way possible to avoid a high negative impact on the system efficiency. The present paper attempts to describe a novel semipassive solar tracking concentrator (SPSTC) in which, in order to track the sun, two independent arrays of polymethyl methacrylate (PMMA) prisms are implemented to refract sunlight by rotating said prisms, thus being able to redirect solar radiation as desired. The first set is responsible for eliminating one of the directional components of the solar radiation; the task is achieved by rotating the prisms within the array at a specific angle. The second set deals with another of the sunlight's directional components, transforming its direction into a completely perpendicular pattern to the array. Having downward vertical radiation makes it possible to implement a stationary Fresnel lens to concentrate the solar radiation for any application desired. The system is designed and validated using simulation software to prove the feasibility of the concept.

\section{Introduction}

Most research efforts on solar energy have focused on photovoltaic (PV) cells development, which generate electricity when a molecular chain reaction that is triggered by a solar energy flux occurs on a thin layer of silicon or germanium based compound. However, commercial PV cells have only achieved an efficiency of $15 \%$ to $20 \%$ so far [1], while high concentration photovoltaic (HCPV) cells have efficiency slightly above $40 \%$ [2].

Another research line explores the use of solar thermal energy (STE), in which case two main issues must be overcome. Firstly, solar radiation must be concentrated for high temperature applications; this is due the low density nature of such energy. Secondly, for most solar concentrators, solar rays must fall perpendicular to the concentrator at all times. Therefore, a solar tracking concentrator (STC) must be used. These requirements also apply to HCPV.

This work was carried on to explore the feasibility of a STC using a semipassive solar tracking device consisting in two arrays of prisms, a Fresnel lens, and a receiver. The arrays track the position of the sun and bend the sun light toward the Fresnel lens, which, as the concentration spot, remains fixed.
The STC can be used in either HCPV or concentrated solar power (CSP) applications.

1.1. Solar Concentration and Tracking. There are two types of solar concentration; they are linear and spot concentrations. Linear or $2 \mathrm{D}$ concentration refers to an area being concentrated to a line and 3D concentration refers to an area being concentrated to a spot. 3D concentration achieves the highest practical concentration level. Solar irradiance intensity varies depending on geographical location, season of the year, weather conditions, and time of day. Liu and Jordan model [3] was used to describe solar irradiance of Monterrey, Mexico (with a latitude of $25^{\circ} 40^{\prime} \mathrm{N}$ ), from where it can be seen that the highest irradiance intensities are between 9:00 hours and 15:00 hours; consequently the semipassive solar tracking concentrator (SPSTC) is designed to work during that period of time.

A wide variety of STC exists which mainly use Fresnel lenses, parabolic mirrors, unconventional geometries, and even lenses with variable optical properties [4-11]. In this paper, a SPSTC that reduces the mechanical effort for sun tracking is presented. It diminishes the system's energy consumption and complexity, therefore reducing system's 


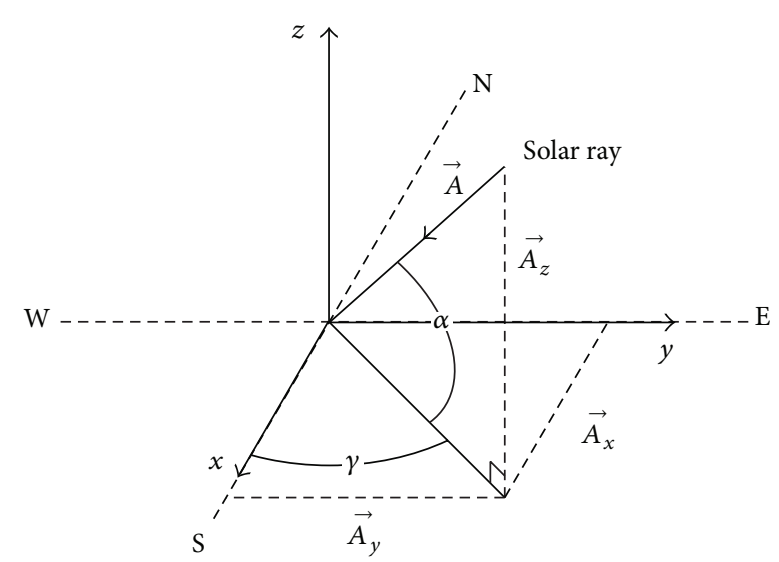

FIGURE 1: Solar angles and direction vector of the solar ray $\vec{A}$ with $x y z$ components and coordinate system.

costs. It consists of a couple of PMMA prism arrays that track the sun's rays and a fixed Fresnel lens that concentrates them.

1.2. Solar Angles. The sun's movement throughout the sky may be described by two angles: altitude and azimuth. The first one is the vertical angle the sun makes with the ground plane, having only the following possible values: $0^{\circ} \leq \alpha \leq 90^{\circ}$. Conventionally, the azimuth is the horizontal angle between the sun and earth's north, but for practical purposes in this study it has been defined using the south as reference being positive in direction toward east (according to the right hand rule): $-180^{\circ}<\gamma<180^{\circ}$ as shown in Figure 1.

The angles depend not only on the time of the day, but on the time of the year as well (see Figure 2), analogous to the irradiance variations. The solar angles are a function of the latitude and can be calculated according to established models [12]. The objective of knowing the solar position is to validate our system in actual possible values of solar angles.

\section{Semipassive Solar Tracking Concentrator (SPSTC)}

The SPSTC is an optic system that concentrates solar radiation with minimal movement requirements while harvesting the maximum possible energy in a certain area. It has two layers of PMMA prisms that redirect the received solar rays in a constant vertical direction toward a Fresnel lens.

Even though the system still requires moving components, it represents an improvement in comparison to conventional tracking and concentrating systems: (i) lower installation and maintenance; (ii) the required mechanism for the tracking is simplified because the only moving components in the system are lightweight prisms, which can be rotated using a single linear actuator for each array, in contrast to more complex structures whose motion implies carrying large weights; (iii) the Fresnel lens, which is the concentrating part of the system, remains in a fixed position; (iv) consequently the receiver does not need to be repositioned; and (v) finally, the wind loads are reduced, since the gap between each prism allows the air to flow freely.
2.1. Prism Arrays. The arrays are located above the Fresnel lens. They are perpendicular to each other and remain horizontal as shown in Figure 3. Each array consists of a set of identical prisms synchronously rotated by a single actuator; however, prisms of first and second array have different apex angles $(\epsilon)$. The rotation mechanism is similar to a window's shutter but instead of blocking the sun's light, they redirect those solar rays by the refraction of the PMMA prisms.

Every prism in the upper array rotates on its own axis, which is parallel to $y$-axis as shown in Figure 4(b). This array is responsible for eliminating one of the solar ray's direction vector components. Figure 4(c) illustrates the interaction between one prism within the first array and an incoming solar ray. Vectors $\overrightarrow{A_{I}}, \overrightarrow{B_{I}}$, and $\overrightarrow{C_{I}}$ shows the change in path of the ray due to refraction upon the prism. The purpose of the prism is to eliminate the $x$-component of the beam, ideally $C_{I, x}=0$.

Afterward, ray $C_{I}$ reaches a prism of the lower array. Analogously every prism in the lower array rotates an angle $\rho_{I I}$ on its own axis, which is parallel to $x$-axis in order to eliminate the $y$-component, ideally $C_{I I, y}=0$. Leaving the resultant beam with a constant direction; perpendicular to the plane $X Y$.

2.2. Fresnel Lens. It is a type of compact lens that is able to concentrate solar energy in a reduced area; it is essentially a chain of prisms, in which each one represents the slope of the lens surface [13]. The function of this optical device in the system proposed is to receive the rays refracted by the two arrays of prisms and concentrate them in a receiver. This concentration is achieved by two important factors: the change in refractive index of the medium through which the beam travels and the geometry of the lens.

\section{Mathematical Model}

3.1. Light Refraction. The angles of the rotating prisms depend on the solar position, which at each given time defines a directional vector as shown in Figure 1. The physical phenomenon involved in this system in both the prisms arrays and the Fresnel lens is known as refraction of the light. Snell's law only applies when we work in the same plane; however when working with solar rays, we find the problem of double angle, which makes the working plane vary constantly. Therefore vector analysis must be performed to find an extrapolation of Snell's law for a 3D environment.

Start with the plane formed by the incident light beam and the normal vector in the point of incident as seen in Figure 5. In that plane the resulting vector after refraction in terms of $\theta_{2}$ is

$$
\widehat{s}_{2}=\left(\sin \theta_{2}\right) \widehat{v}+\left(\cos \theta_{2}\right) \widehat{N}_{2} .
$$

With basic trigonometry

$$
\cos \theta_{2}=\sqrt{1-\sin ^{2} \theta_{2}} .
$$

On the other hand Snell's law states

$$
\sin \theta_{2}=\frac{n_{1}}{n_{2}} \sin \theta_{1} .
$$




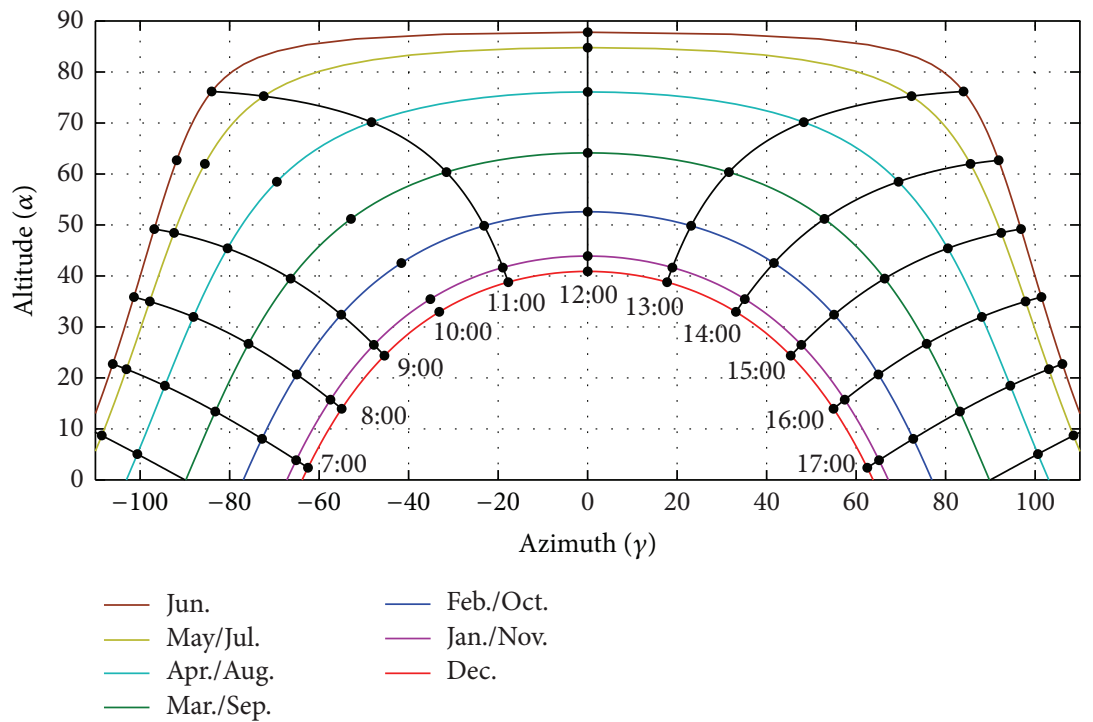

FIGURE 2: Altitude and azimuth throughout the year.

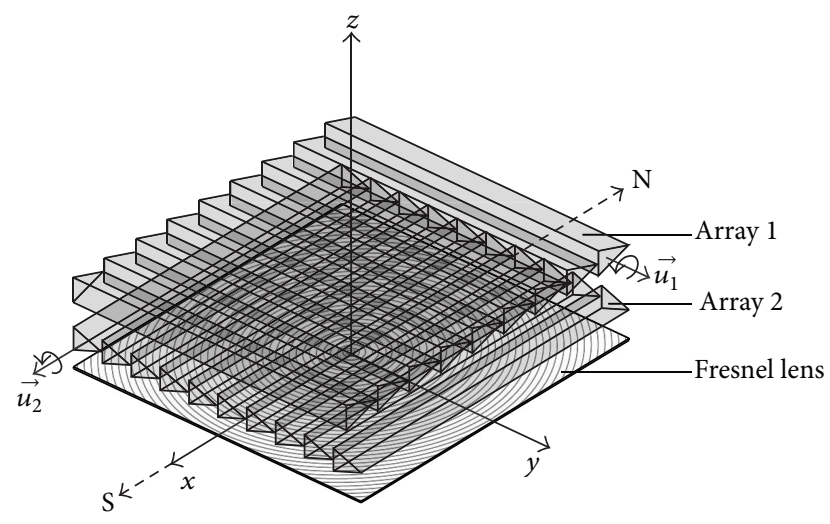

FIGURE 3: SPSTC formed by two prisms arrays and a Fresnel lens.

Substituting (2) and (3) onto (1) the following expression is obtained:

$$
\widehat{s}_{2}=\left(\frac{n_{1}}{n_{2}} \sin \theta_{1}\right) \widehat{v}-\widehat{N} \sqrt{1-\left(\frac{n_{1}}{n_{2}}\right)^{2} \sin ^{2} \theta_{1}} .
$$

Equation (4) is a good approach; notice that in space the cross product of unitary vectors $\widehat{N}_{1}$ and $\widehat{s}_{1}$ is

$$
\widehat{N}_{1} \times \widehat{s}_{1}=\left|\widehat{N}_{1}\right| \cdot\left|\widehat{s}_{1}\right| \sin \theta_{1} \cdot \widehat{u}=\sin \theta_{1} \cdot \widehat{u},
$$

where $\widehat{u}_{1}$ is a unitary vector normal to the initial plane, obeying the right hand rule, as shown in Figure 6(b). A clever manipulation using dot product in expression (5) yields the following:

$$
\sin ^{2} \theta_{1}=\left(\widehat{N} \times \widehat{s}_{1}\right) \cdot\left(\widehat{N} \times \widehat{s}_{1}\right) .
$$

Remember $\widehat{v}$ is normal to the plane defined by $\widehat{N}_{1}$ and $\widehat{u}$; therefore

$$
\widehat{v}=\widehat{N}_{1} \times-\widehat{u} .
$$

Merging (5) with (7)

$$
\widehat{v}=\frac{1}{\sin \theta_{1}} \widehat{N} \times\left(-\widehat{N} \times \widehat{s}_{1}\right) .
$$

Finally, substituting (6) and (8) in (4) an extrapolation of Snell's Law is obtained, in function of vectors $\widehat{s}_{1}$ and $\widehat{N}$ only, which are the incident ray and the normal to the plane, respectively,

$$
\begin{aligned}
\widehat{s}_{2}= & \frac{n_{1}}{n_{2}} \widehat{N} \times\left(-\widehat{N} \times \widehat{s}_{1}\right) \\
& -\widehat{N} \sqrt{1-\left(\frac{n_{1}}{n_{2}}\right)^{2}\left(\widehat{N} \times \widehat{s}_{1}\right) \cdot\left(\widehat{N} \times \widehat{s}_{1}\right)} .
\end{aligned}
$$

In order to exploit (9), the normal vector must be calculated for both cases when the ray is entering the prism, as for when it is going out of it. Both of them depend on the rotation of the prism $\rho$, as it would be expected and is appreciated in Figure 6. But $\epsilon$ once it is defined it will not change throughout the operation of the system.

Given values $\alpha, \gamma$, the direction vector of the solar ray is defined by

$$
\overrightarrow{A_{I}}=[-\cos \alpha * \cos \gamma,-\cos \alpha * \sin \gamma,-\sin \alpha] .
$$

For every angle $\rho$, everything else is determined; therefore the path that a solar ray traces is unique. First with (9) and $\vec{A}$ we obtain the ray inside the prism: $\vec{B}$; then with $\vec{B}$ and (9) $\vec{C}$ can be found. Once the analysis is complete for the first array of prisms, it follows the same way for the second set.

\section{Solar Tracking by Rotating Prism Array}

4.1. First Layer of Prisms. With the help of Matlab, the behavior of a ray through the prism for all possible rotations may be 


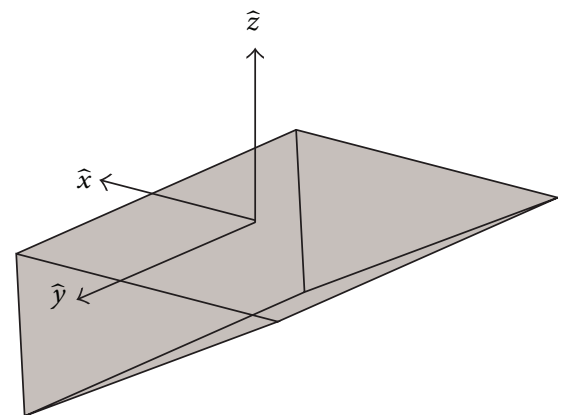

(a)

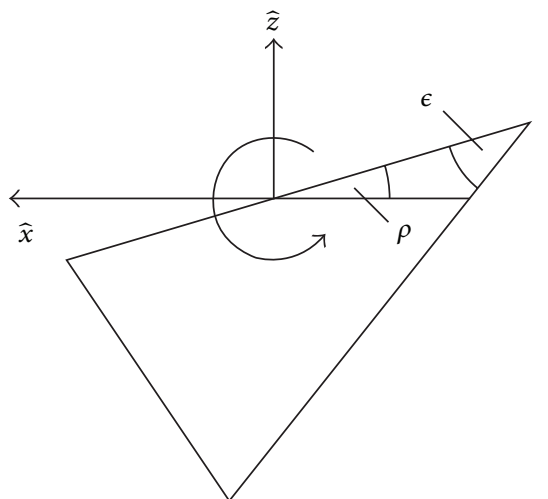

(b)

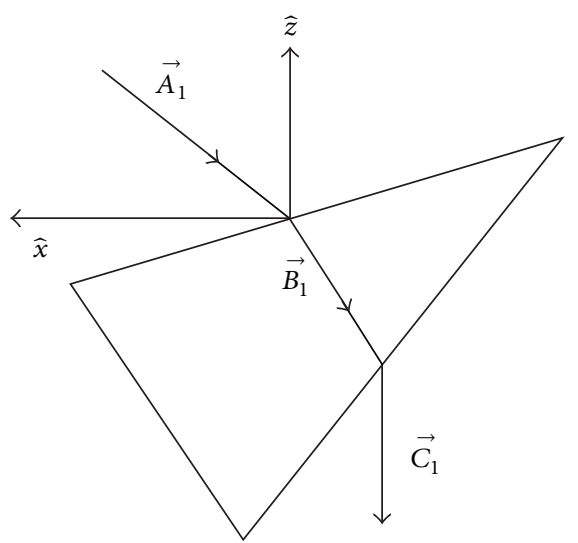

(c)

FIgURE 4: (a) Spatial orientation of a prism from 1st array. (b) Prism rotated $\rho_{I}$. (c) Ray's path through prism 1.

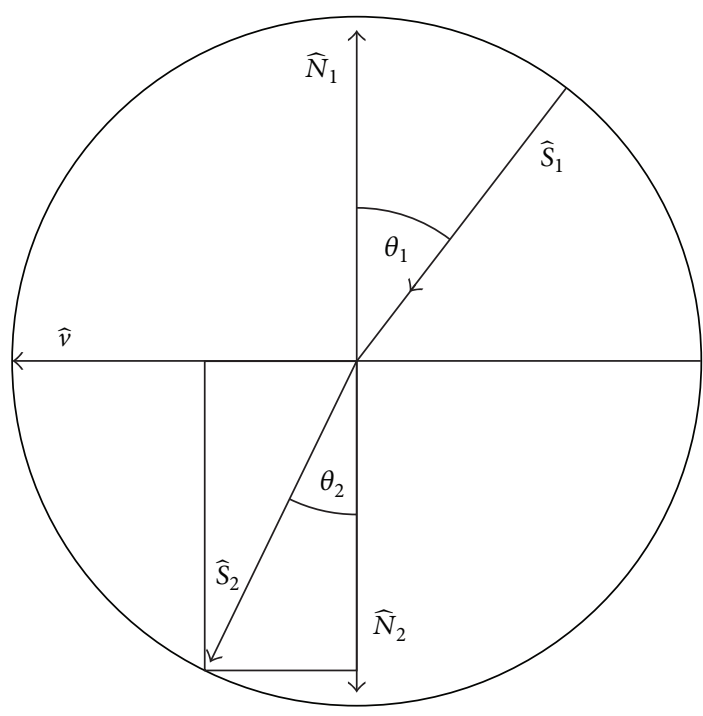

(a)

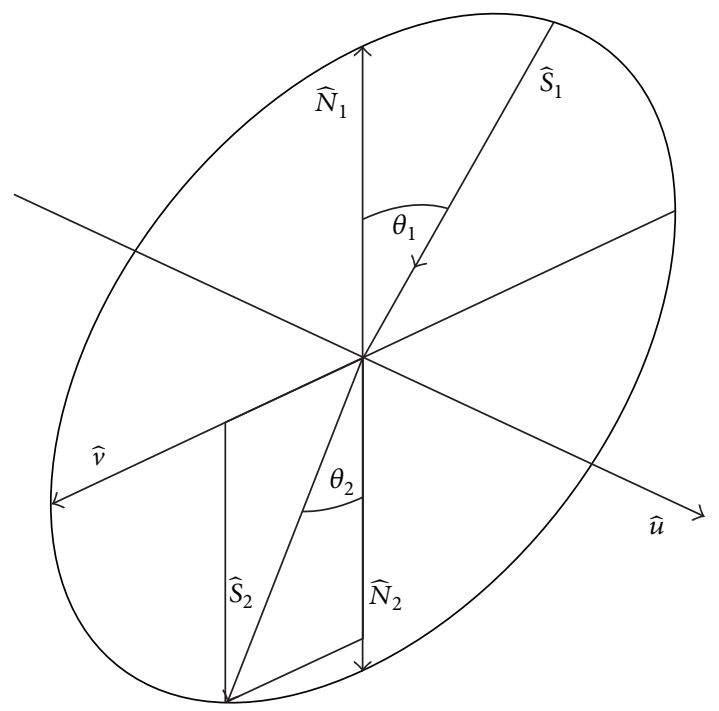

(b)

Figure 5: (a) Snell's law in a plane; (b) Snell's law in space. 


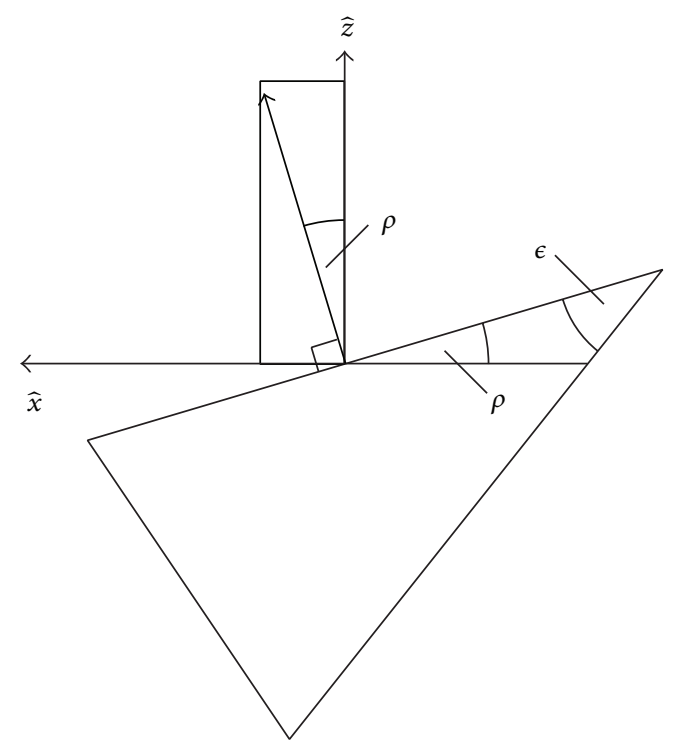

(a)

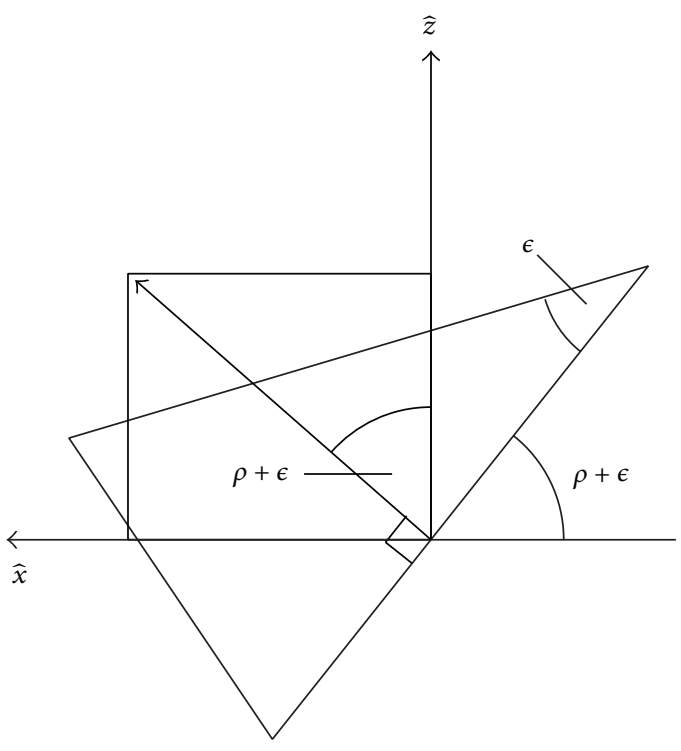

(b)

FiguRE 6: (a) Normal vector for rays entering the prism; (b) normal vector for rays leaving the prism.

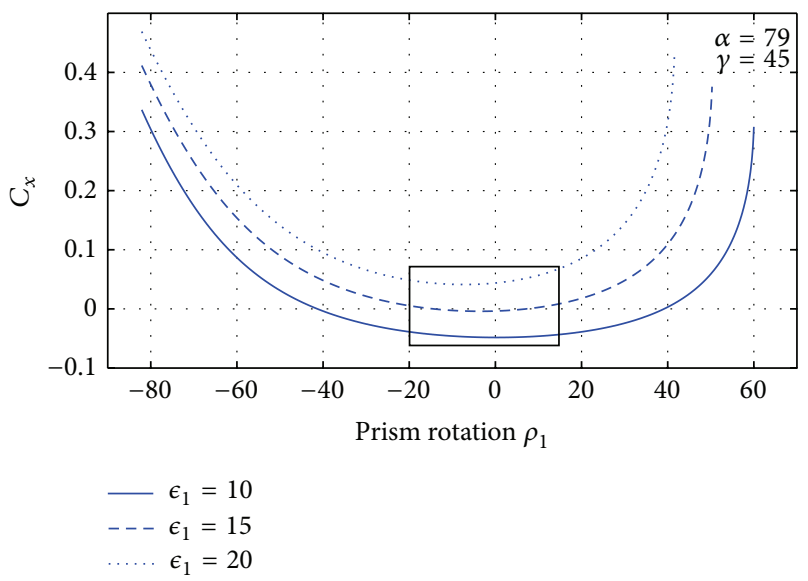

(a)

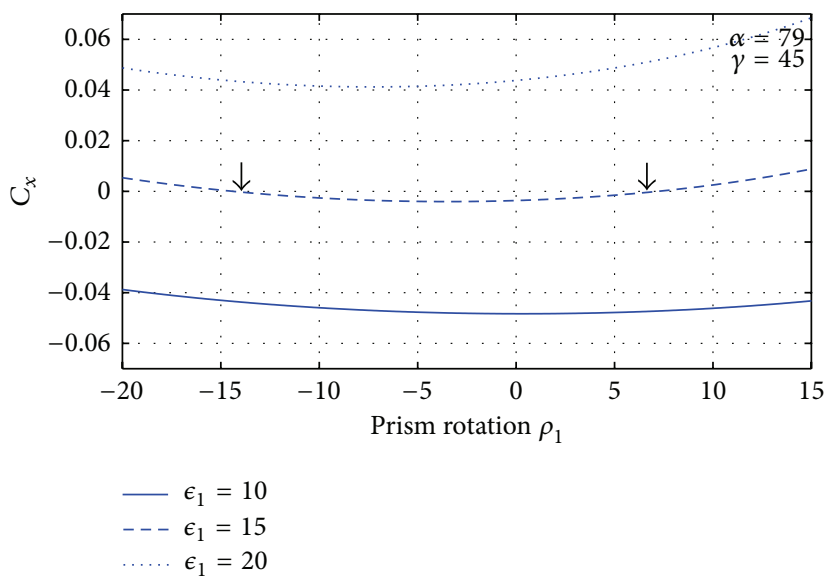

(b)

Figure 7: (a) Output response $C_{x}$ for prism rotating $\rho_{1}$ for distinct values of $\epsilon_{1}$; (b) zoom view.

obtained. The component $C_{x}$ is of interest, since the purpose of the first prisms array is to eliminate such component.

It may be observed in Figure 7 that for a particular $\alpha$ and $\gamma$ if $\epsilon$ increases the system will not be able to eliminate the desired component $x$; meanwhile when it does, there are two values $\rho$ that accomplish the desired value. But if $\epsilon$ decreases too much, even though they still have two possible solutions, remembering that if the prism has a bigger rotation (positive or negative) then the area available to collect the sun rays will be smaller. Therefore, an adequate value of $\epsilon$ that ensures $C_{x}=$ 0 and minimizes the $|\rho|$ throughout the day and along the months must be chosen.

4.2. Second Layer of Prisms. Using exactly the same analysis as above, instead of having an incoming solar ray $A_{I}$, the ray comes from the previous prism array and therefore has a known direction $C_{I}$. In other words it holds that incoming vector

$$
\overrightarrow{A_{I I}}=\overrightarrow{C_{I}}
$$

An important remark is that the analysis in Section 4.1 seeks to eliminate the $x$-component, then $\overrightarrow{C_{I, x}}=0$; therefore, the path through the prism in the second array, described by the vectors $\overrightarrow{A_{I I}}, \overrightarrow{B_{I I}}, \overrightarrow{C_{I I}}$, will all be in the YZ-plane (see Figure $8(\mathrm{a})$ ) and the goal of the second set of prisms will be to eliminate the $y$-component, obtaining $C_{I I, y}=0$. When the ray travels through the same plane $Y Z$, (3) may be used to determine the path without the use of vector; this analysis is similar to the analysis necessary for designing a Fresnel lens and is well described in [3]; however the ideal value $C_{I, x}=0$ may not be always possible; therefore the goal of both sets 


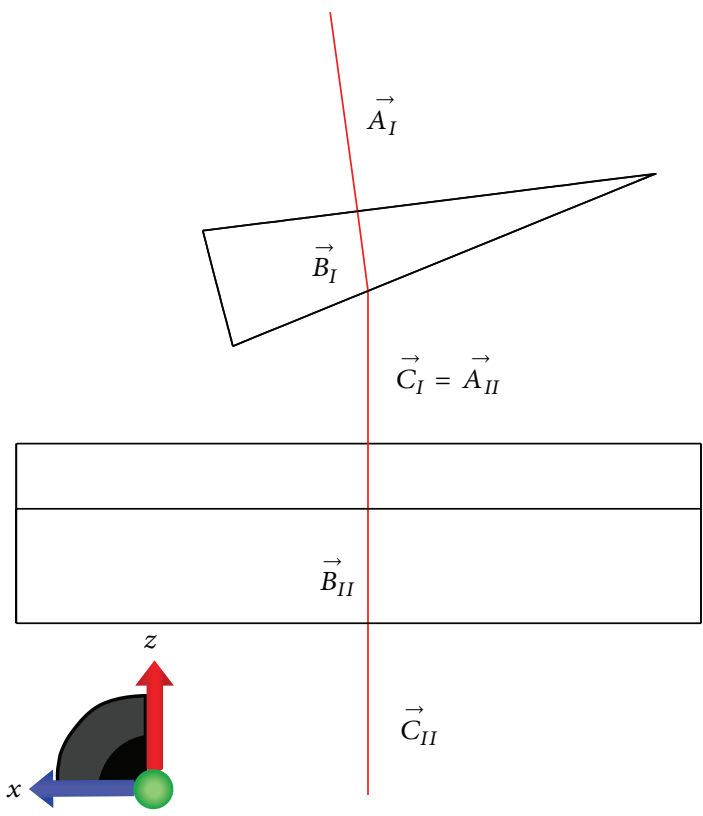

(a)

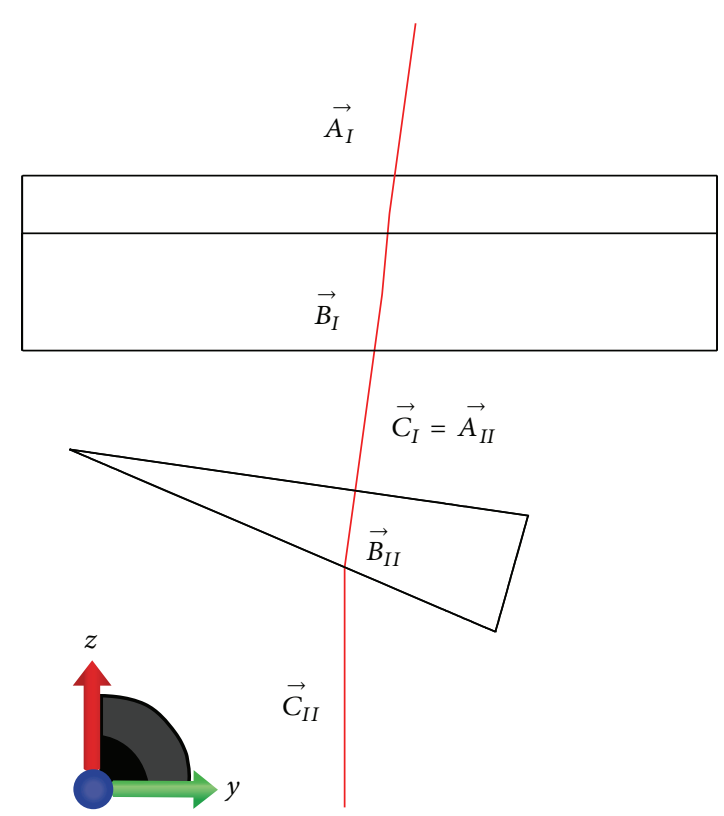

(b)

FIGURE 8: Solar path through prisms $I$ and $I I$ : (a) front view; (b) lateral view.

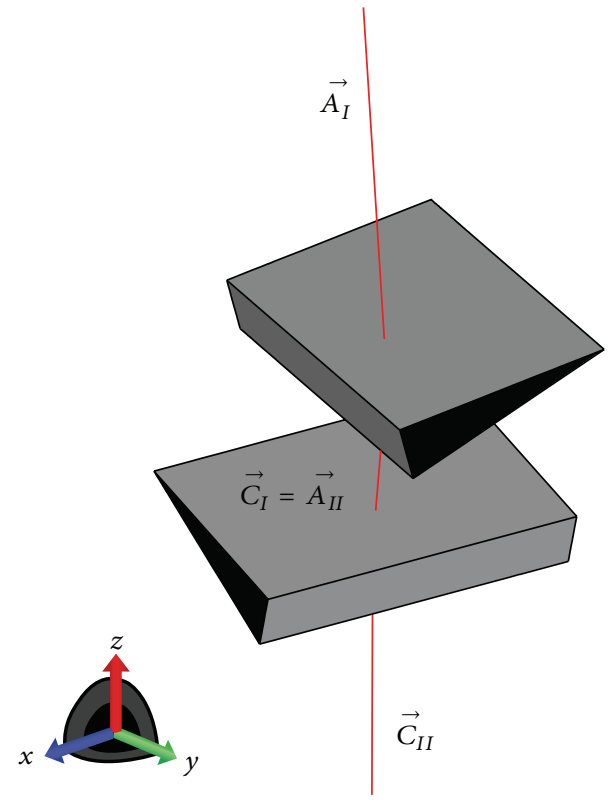

FIGURE 9: Solar path through prisms $I$ and $I I$ : isometric view.

of prisms is to minimize the respective components so the Fresnel lens can operate efficiently.

\section{Computer Simulation}

After having obtained values with Matlab, the model is evaluated using TracePro in order to verify the trajectory the solar rays will follow corroborating the results predicted in the calculations. It also helps to have a better understanding of the behavior of the rays in a 3D environment. For instance, in Figure $8(\mathrm{~b})$ it can be observed how $\overrightarrow{C_{I}}$ has a $y$-component which will be dealt with by the lower prism.

Additionally to the simulation shown in Figure 8 of just one pair of prisms and one solar ray, the whole system can be visualized in Figure 9 for a given time of the day $\left(\alpha=79^{\circ}\right.$, $\gamma=45^{\circ}$ ), where the concentration spot formed by the Fresnel lens is shown.

\section{System's Parameters}

In Figure 10 the system can be seen working for a particular point in time, and the process is shown for given arbitrary fixed parameters of the system of how to obtain working values $\rho_{1}, \rho_{2}$ to achieve the correct redirection of solar rays. It is important to understand and visualize all the design parameters that must be determined.

6.1. Prisms Inner Angle ( $\epsilon)$. One key parameter of design is the angle within the prism. In typical Fresnel lenses using simple refraction of light, angles along the profile that are further away from the center will increase until certain critical angle which is defined by the mediums refractive coefficients and restricted by Snell's Law. In the system proposed these angles not only are responsible for the whole optic response of the system, but will later be a factor on the thickness of the prisms itself.

6.2. Prism's Rotation Axis $(\widehat{u})$. The process shown assumes rotation vectors matching the coordinate system as seen in Figure 4 for the upper layer. These may not be ideal and despite the fact that we can have any direction, the symmetry of the sun's movement within a day can be still harnessed with 

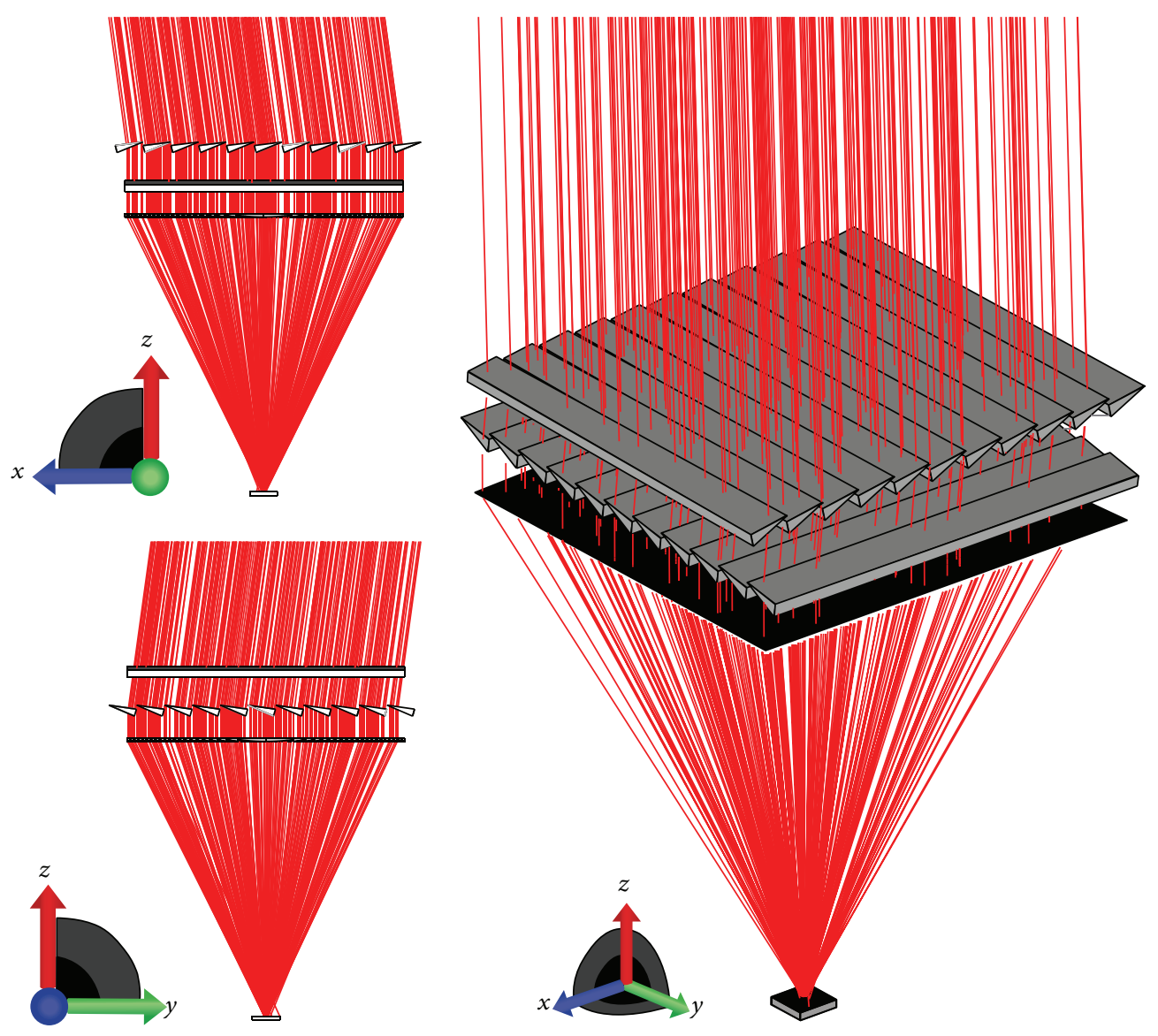

Figure 10: TracePro simulation for $\alpha=79^{\circ}, \gamma=45^{\circ}$.

a slight elevation of the original proposed rotation axis by a $\phi$ angle above the $X Y$ plane. The resulting rotation axis

$$
\widehat{u}_{1}=[\cos \phi, 0,-\sin \phi] \text {. }
$$

6.3. Solar Ray Output $\left(\overrightarrow{C_{I I}}\right)$. The advantage of a fixed Fresnel lens in the system does not restrict the direction of the output rays; therefore the vertical direction previously described can be defined to any direction that helps the overall system. Similar to the previous section the symmetry can be exploited by a slight deviation of $\delta$ degrees, without fixing the idea to an arbitrary vertical output. This means the Fresnel lens must have a slight elevation resulting in the next directional vector for the solar rays:

$$
\overrightarrow{C_{I I}}=[-\sin \delta, 0,-\cos \delta] .
$$

\section{System's Admittance Rays}

The previous parameters must be defined according to the system overall efficiency; therefore a validation parameter must be placed to determine which combination offers better results.

7.1. Snell's Law as a Bijective Function. Snell law (see (3)) can be used to determine the relation between the angle of incidence and refraction; however there is a one-to-one correspondence between these angles once the mediums are fixed. The main reason is that sine function is bijective with $\theta \in[0, \pi / 2]$, and respective refractive indexes and normal vector are the same:

$$
\sin \theta_{1}=\frac{n_{2}}{n_{1}} \sin \theta_{2}
$$

Therefore from (3) we can obtain (14) and Snell law is also satisfied if the incident angle is in medium 2 and refractive angle in medium 1 as shown in Figure 11.

7.2. Working Backwards. Ideally a solar ray inputs the system and changes direction outputs in a fixed direction, but a different approach may be taken: given certain system parameters all possible paths of a ray coming in the opposite direction of desired output $\overrightarrow{C_{I I}}$ can be obtained by rotating both prisms in all possible $\rho$ values (see Figure 12). For every combination of $\rho_{1}, \rho_{2}$ a point is plotted to obtain a scatter graph that represents the system's admittance rays (see Figure 13).

\section{Performance Evaluation}

In order to evaluate the system performance with certain parameters we must acknowledge that the system is desired to work only in a particular window of time. In Figure 2 

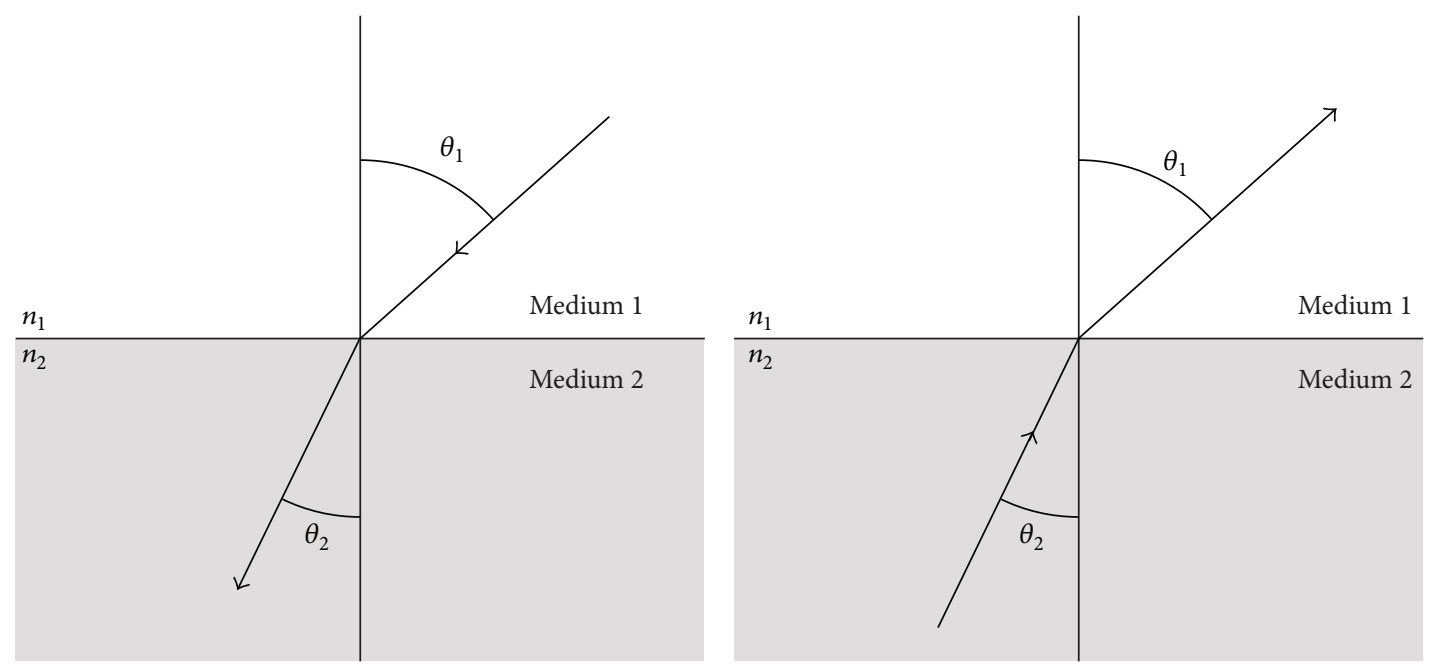

FIGURE 11: Snell's law as a bijective function.

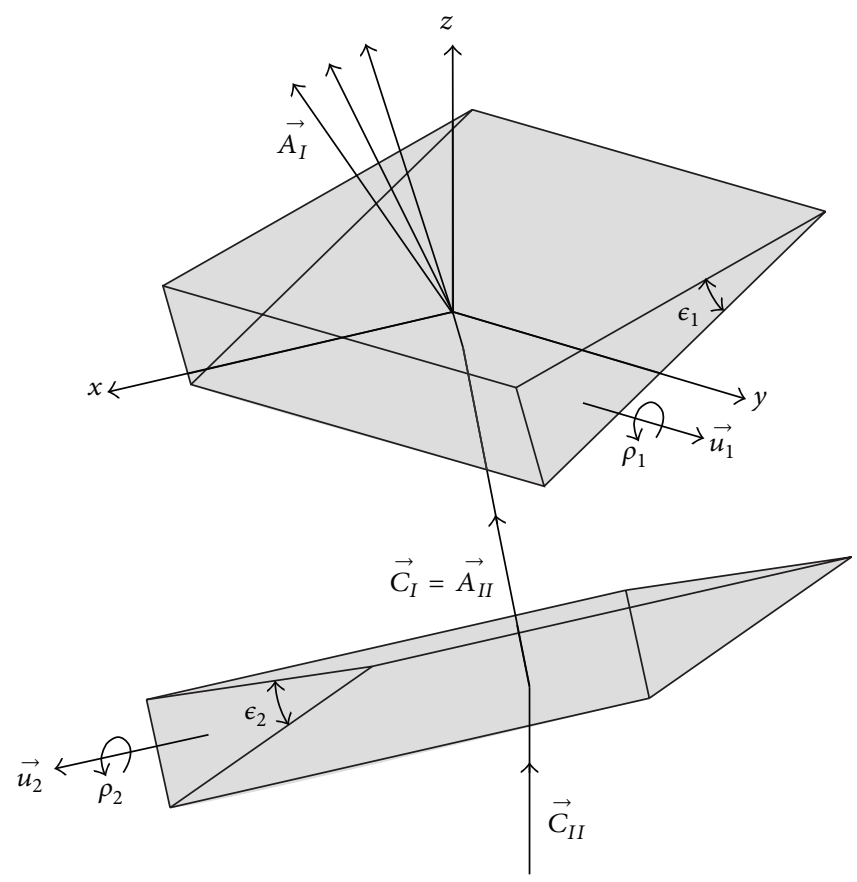

FIGURE 12: Reverse path of solar rays in a system with fixed $\epsilon_{1}, \epsilon_{2}, \overrightarrow{u_{1}}, \overrightarrow{u_{2}}$, and $\overrightarrow{C_{I I}}$.

all possible values of solar angles are shown throughout the year but a more realistic and achievable goal is to capture the sunlight on a certain schedule, for instance, from 9:00 to 15:00 hrs delimiting the region shown in Figure 14.

Then working backwards for a system with defined parameters, the system's admittance angles are obtained and compared to the desired area as shown in Figure 15.

The points that fall outside the domain are not of our interest, since there will never be a time of the year with such angular values. Therefore as seen in Figure 16, the blue area is a valid parameter to evaluate the system performance.

\section{Conclusions and Future Work}

It has been theoretically proven that a SPSTC that uses exclusively PMMA prisms rotating according to the sun's position is feasible. The current implementation has a fixed Fresnel lens which reduces greatly the mechanical effort of the system, since the tracking is performed by the rotating prisms arrays, which have the virtue of being lightweight individual prisms instead of a robust big heliostat that has to move in a 3D manner. A primary virtue of the system is that every prism within an array rotates the same angle; therefore the movement mechanism can be simplified. 


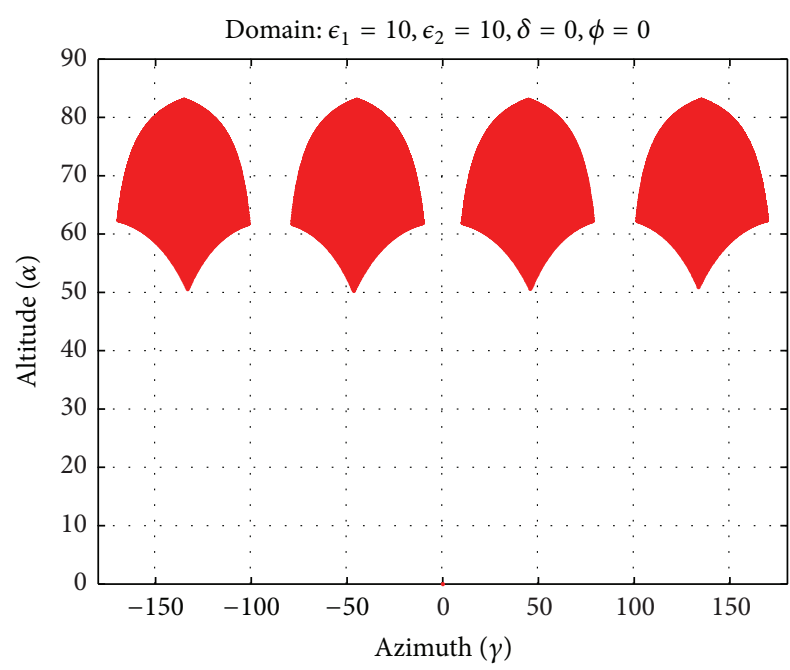

Figure 13: Domain for $\epsilon_{1}=10^{\circ}, \epsilon_{2}=10^{\circ}$, and $\overrightarrow{C_{I I}}=[0,0,-1]$.

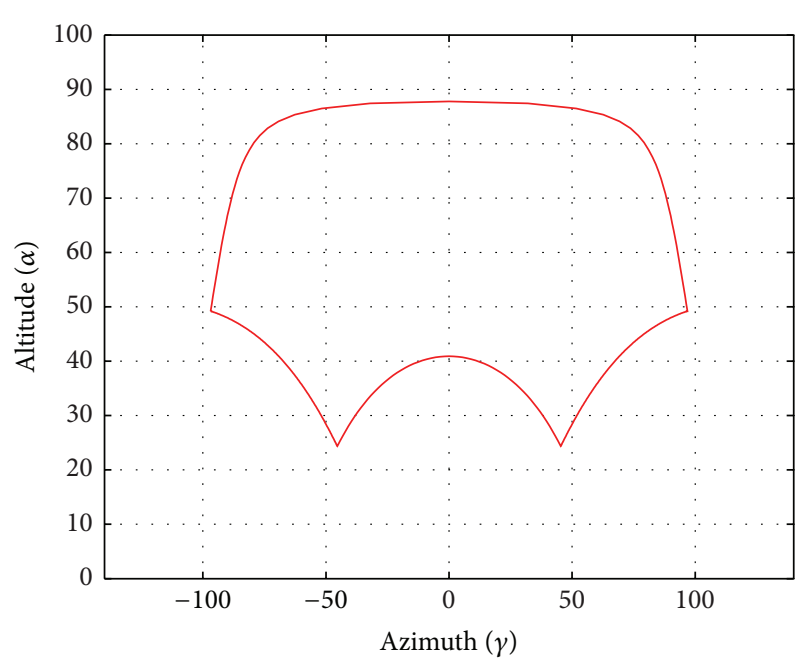

FIGURE 14: Desired domain of the system.

The tracking is achieved by separating the dual axis movement and implementing two independent rotational movements that work hand in hand to refract efficiently solar rays. Doing so offers a simpler approach in tracking the sun which ultimately would result in cheaper but reliable solution.

Further analysis must be done to find the optimal values of $\epsilon_{I}, \epsilon_{I I}, \phi$, and $\delta$ that manage to operate within the desired time frame.

\section{Nomenclature}

$\vec{A}: \quad$ Ray direction vector incident to the prism

$\vec{B}$ : $\quad$ Ray direction vector within the prism

$\vec{C}$ : $\quad$ Ray direction vector outside the prism

$\vec{u}$ : $\quad$ Prism's rotation axis

$n$ : $\quad$ Refractive index (-)

PMMA: Polymethyl methacrylate

$I_{\text {dir }}$ : Direct solar irradiance $\left(\mathrm{W} / \mathrm{m}^{2}\right)$.

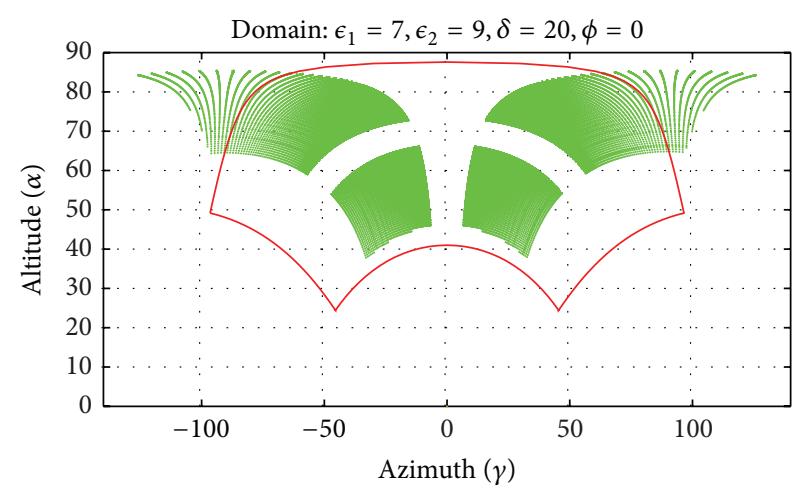

FIGURE 15: Domain of the system for $\epsilon_{1}=7, \epsilon_{2}=9, \delta=20$, and $\phi=0$.

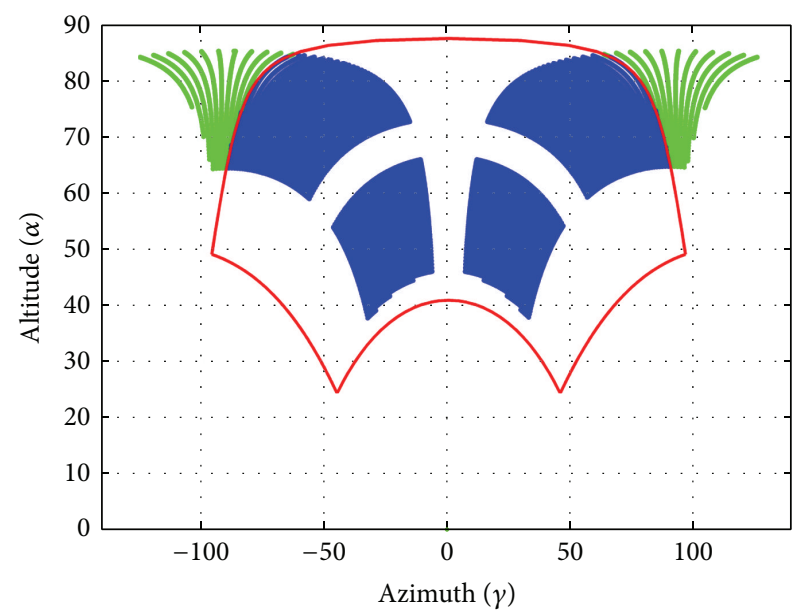

FIGURE 16: Valid domain of the system for $\epsilon_{1}=7, \epsilon_{2}=9, \delta=$ 20 , and $\phi=0$.

\section{Greek Symbols}

$\alpha$ : Solar altitude $\left(^{\circ}\right)$

$\gamma$ : Solar azimuth $\left({ }^{\circ}\right)$

$\epsilon$ : Prism's inner angle $\left({ }^{\circ}\right)$

$\rho$ : Prism's rotation angle $\left({ }^{\circ}\right)$

$\phi$ : Array's rotation above plane $x y\left({ }^{\circ}\right)$

$\delta$ : Output vector "altitude" $\left({ }^{\circ}\right)$.

\section{Subscripts}

$x: x$-vector component

$y: \quad y$-vector component

$z: \quad z$-vector component

$I: \quad 1$ st array of prisms

$I I$ : 2nd array of prisms.

\section{Conflict of Interests}

The authors declare that there is no conflict of interests regarding the publication of this paper. 


\section{Acknowledgments}

The authors acknowledge Lambda Research for supporting research aimed to help human development and for the licensed copy of TracePro, Autodesk for donating free software licenses to ITESM students, and the Research Chair of Design and Innovation in Engineering: "Solar Thermal Vehicle (STEV)" for directly supporting this work.

\section{References}

[1] M. Green, K. Emery, Y. Hishikawa, and W. Warta, "Progress in photovoltaics: research and applications solar," Cell Efficiency Tables, vol. 17, no. 5, pp. 320-326, 2009.

[2] P. Pérez-Higueras, E. Muñoz, G. Almonacid, and P. G. Vidal, "High Concentrator PhotoVoltaics efficiencies: present status and forecast," Renewable and Sustainable Energy Reviews, vol. 15, no. 4, pp. 1810-1815, 2011.

[3] B. Y. H. Liu and R. C. Jordan, "The interrelationship and characteristic distribution of direct, diffuse and total solar radiation," Solar Energy, vol. 4, no. 3, pp. 1-19, 1960.

[4] S. Barone, “Directed Fresnel lenses," US Patent 2005/0041307A1, 2005.

[5] K. Johnson, "Fresnel scroll solar tracking Device," US Patent 4,765,726, 1988.

[6] J. Baker, "Multielement optical panel," US Patent 4,456,783, 1984.

[7] A. B. Larbi, "A new design of a (3D) fresnel collector with fixed mirrors and tracking absorber," Journal of Solar Energy Engineering, Transactions of the ASME, vol. 122, no. 2, pp. 63-68, 2000.

[8] R. Gorthala, "Method and apparatus for a passive solar day lighting system," US Patent 6,299,317B1, 2001.

[9] J. Pender, "Motion-free tracking solar concentrator," US Patent 6,958,868, 2005.

[10] "Method and system for light ray concentration," patent no. US2009/0250094, assignee, SolBeam Inc, 2009.

[11] D. Chemisana, Diseño y caracterización de un concentrador térmico-fotovoltaico cuasiestacionario para integración arquitectónica [Ph.D. thesis], Universitat Lleida, 2009.

[12] J. Duffie and A. William, Solar Engineering of Thermal Processes, John Wiley \& Sons, New York, NY, USA, 2nd edition, 1991.

[13] R. Leutz and A. Suzuki, Non-Imaging Fresnel Lenses: Design and Performance of Solar Concentrators, Springer, 2001. 

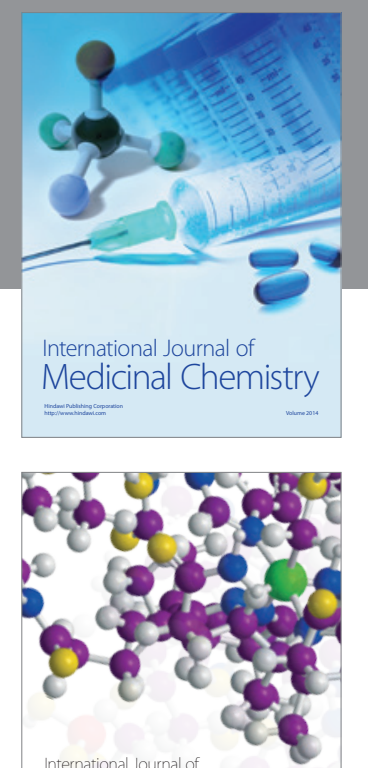

\section{Carbohydrate} Chemistry

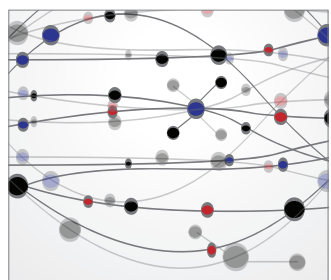

The Scientific World Journal
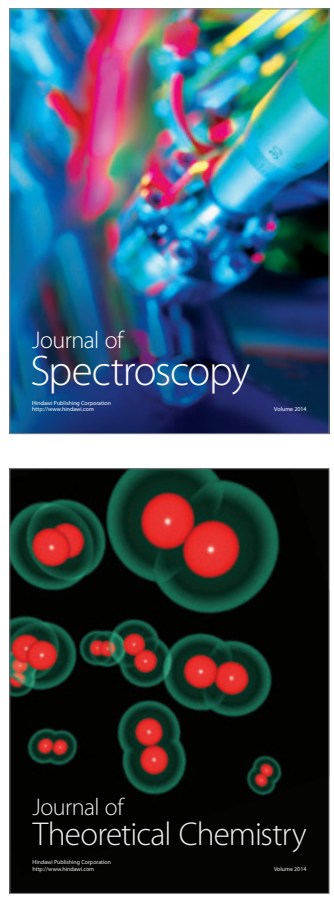
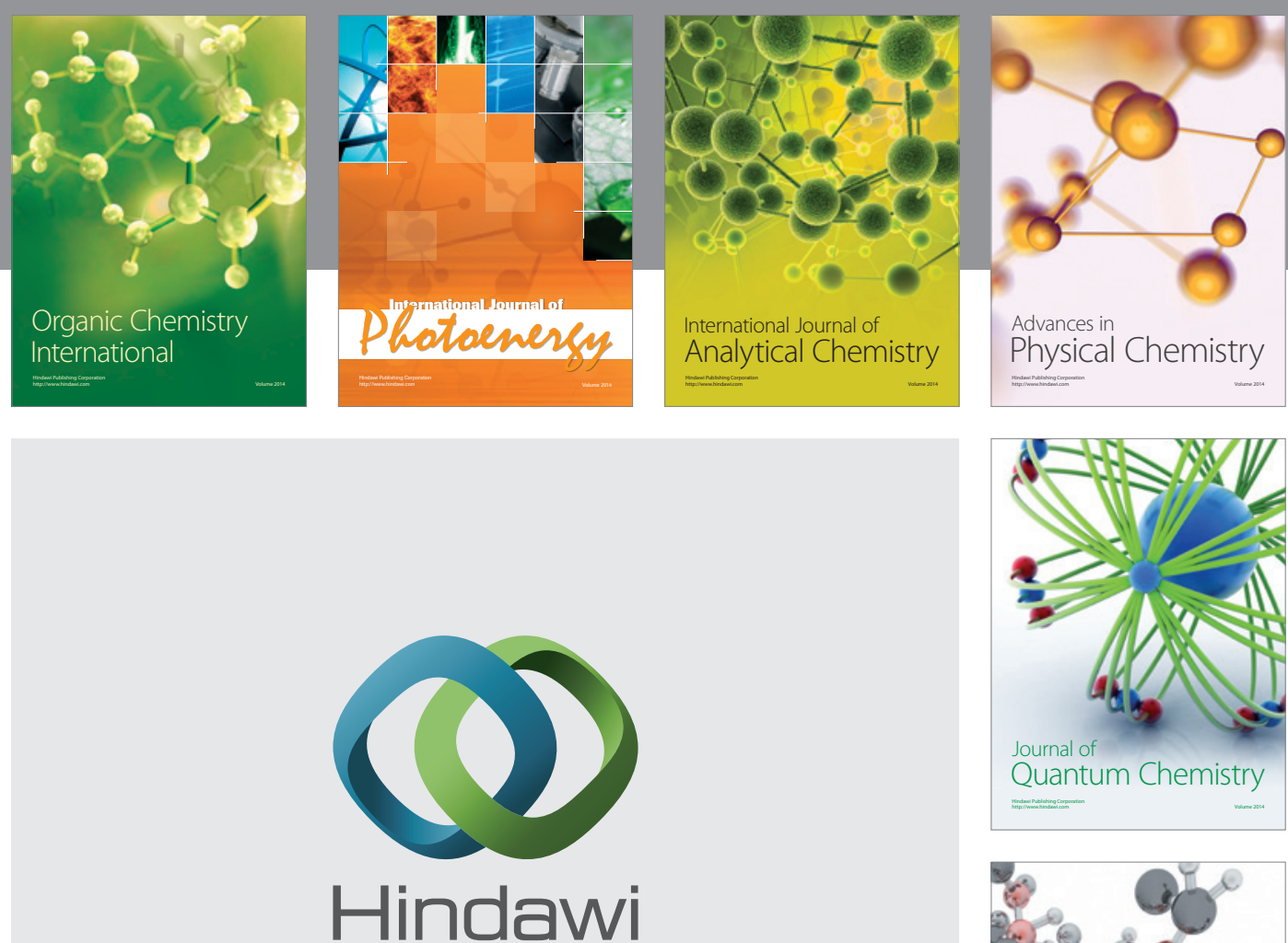

Submit your manuscripts at

http://www.hindawi.com

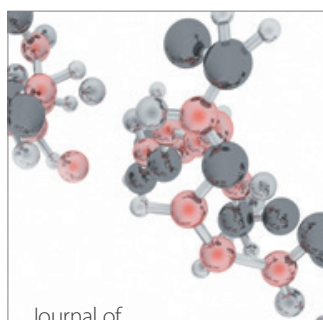

Analytical Methods

in Chemistry

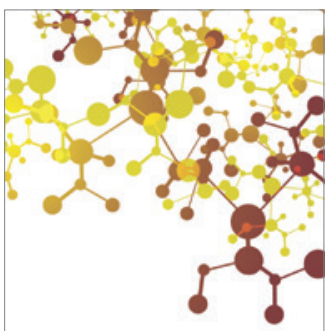

Journal of

Applied Chemistry

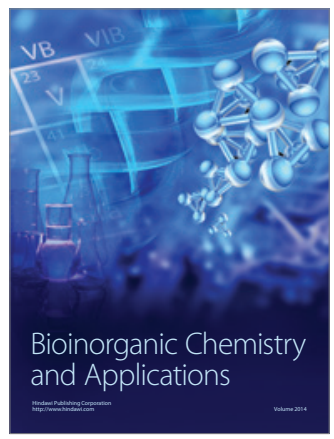

Inorganic Chemistry
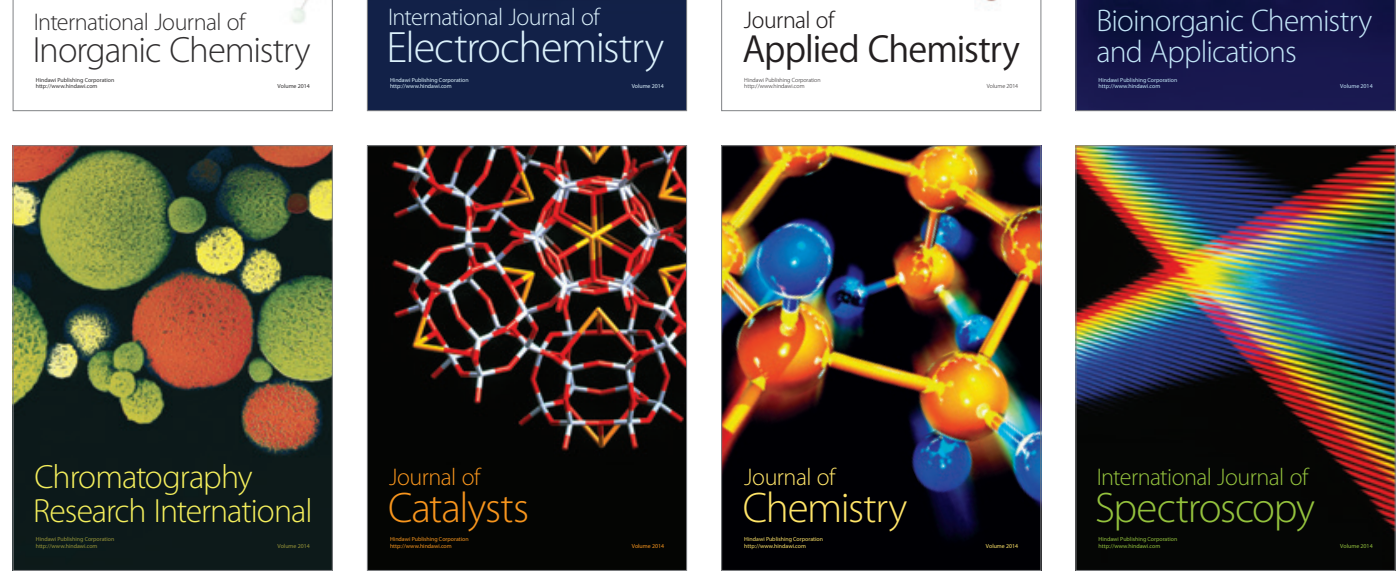